\title{
Application of Parameter-Dependent Robust Control Synthesis to Turbofan Engines
}

\author{
Gregory Wolodkin, Gary J. Balas, and William L. Garrard \\ University of Minnesota, Minneapolis, Minnesota 55455
}

\begin{abstract}
Recent results in multivariable robust control synthesis for linear parameter-varying (LPV) systems are applied to the control of a turbofan engine over a wide range of power codes. Seven linear, time-invariant models are used in the control design. The resulting LPV controller consists of seven linear controllers, gain scheduled via linear interpolation. This gain-scheduled controller is obtained directly as part of the described design process, as opposed to conventional processes, where the gain schedules are developed after the fact to connect point designs. A model matching approach is employed such that the resulting closed loop resembles a decoupled set of secondorder systems with specified rise times and overshoots. The performance of linear $\mathcal{H}_{\infty}$ point designs are compared with the LPV controller at fixed operating points. A nonlinear simulation is performed with the turbofan engine and LPV controller schedules as a function of the power code. The LPV controller exhibits excellent tracking of reference commands as the power code varies in time.
\end{abstract}

\section{Introduction}

$\mathbf{T}$ ODAY'S turbofan engines use single input/single output controllers to regulate fan speed and various pressure ratios. With the introduction of complex, variable cycle engines, highperformance applications, such as short takeoff and vertical landing engines and the development of new power systems for the highspeed civil transport, provide the impetus to investigate advanced control techniques to optimize the robustness and performance of these multivariable systems. ${ }^{1-3}$
A variety of multivariable control techniques have been applied to aircraft turbofan engines. In most cases, the goal is to regulate engine variables such as fan speed and various pressure ratios using control inputs such as fuel flow, nozzle and duct area, and vane angle. These inner-loop controllers are typically gain-scheduledlinear controllers, sometimes combined with an antiwindup scheme to defend against actuator saturation. Reference 4 provides an extensive reference list to previous work in this area. A more recent case study comparing various robust control techniquescan be found in Ref. 5.
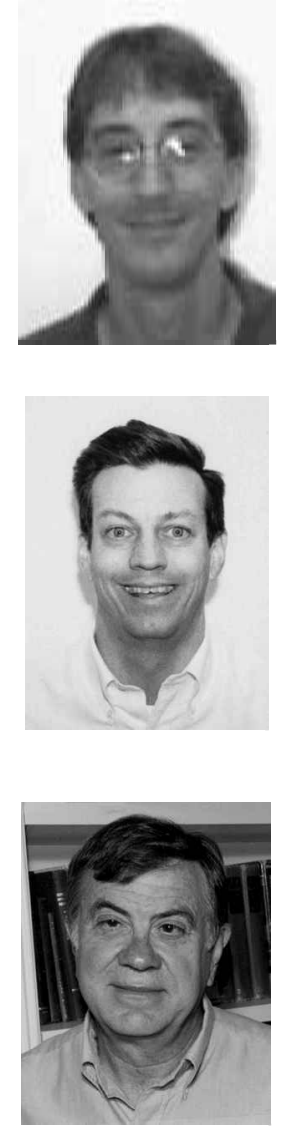

Gregory Wolodkin received his B.S. degree in physics from Frostburg State University, B.S. and M.S. degrees in electrical engineering from the University of Maryland, College Park, and Ph.D. degree in electrical engineering from the University of California, Berkeley, in 1996. Gregory Wolodkin spent the 1996-1997 academic year as a faculty member in the Department of Aerospace Engineering and Mechanics at the University of Minnesota. Currently, he is a senior technical specialist in the Controls group at The MathWorks, Inc., where his interests include system identification, robust control, and parameter estimation for structured nonlinear systems.

Gary J. Balas received his B.S. and M.S. degrees in civil and electrical engineering from University of California, Irvine, and Ph.D. degree in aeronautics and astronautics from the California Institute of Technology in 1990. Since 1990 he has been a faculty member in the Department of Aerospace Engineering and Mechanics at the University of Minnesota. From 1993 to 1995, he held a McKnight-Land Grant Professorship. Currently, Gary Balas is an Associate Professor and Codirector of the Control Science and Dynamical Systems Department at the University of Minnesota and a Senior Member of the AIAA. He is a co-organizer and developer of the MUSYN Robust Control Short Course and the $\mu$-Analysis and Synthesis Toolbox used with MATLAB ${ }^{\circledR}$ and the president of MUSYN, Inc. His research interests include control of flexible structures, aircraft, model validation, and industrial applications of robust control methods.

William L. Garrard received his B.S. in mechanical engineering in 1962 and his Ph.D. in engineering mechanics in 1968, both from the University of Texas, Austin. He is Professor and Head of the Department of Aerospace Engineering at the University of Minnesota. William Garrard is an Associate Fellow of the AIAA and currently serves on the Aerodynamic Decelerator Systems Technical Committee. The work of his team in aerodynamic decelerators resulted in his department and the Army High Performance Computing Research Center of the University of Minnesota being awarded the $\mathbf{1 9 9 6}$ Educational Award for Excellence from the U.S. Army Soldier Systems Command for collaboration between academia and the Army. His research interests are in control of aerospace vehicles and parachute system dynamics. 
The control of nonlinear phenomena such as surge and stall is generally performed by an outer-loop controller. This high-level controller strives to maintain desirable operating conditions (in terms of efficiency, stall margin, etc.) through specification of spool speeds and pressure ratios. In some sense, the high-level controller selects the operating point, and the low-levelor inner-loopcontroller minimizes the tracking error between actual and desired values of spool speeds and pressure ratios. This paper focuses on the design of an inner-loopcontrollerfor an aircraft turbofanengine. It is assumed that the engine inner-loop controller can be designed independently from the outer-loop controller. This assumption is valid when there is approximately a factor of four separation between the inner-loop and the outer-loop control bandwidths.

The turbofan industry and NASA have taken an active research role in the application of multivariable control techniques to aircraft engines. General Electric Aircraft Engine has approached multivariable control using Edmunds model matching design technique. ${ }^{6}$ This method allows the designer to specify a second-order, decoupled response of the individual channels with a fixed structure controller. Because the control design method is performed only at specific frequency points, there is no guarantee that the closed-loop system will be stable, much less stable in the presence of modeling errors. ${ }^{7}$ Linear, point designs are performed at operating points, and the gains are scheduled either via a lookup table or curve fits ${ }^{8}$ to operate across the flight envelope. This scheduling approach also has no guarantee with regard to the stability and performance of the scheduled design

To address stability and performance issues for multivariable engine control design, $\mathcal{H}_{\infty}$ synthesis techniques have been applied.? One approach involves the design of a low-order, robust $\mathcal{H}_{\infty}$ controller that is scheduled as a function of power code. ${ }^{3}$ The controller is scheduled via a constant scaling matrix on the output of the controller. An optimization problem is formulated to find the optimal scheduling matrix. An antiwindup scheme is added to the design to address control saturation limits. As with the previous technique, there are no guarantees with regard to the stability and performance of the scheduled design.

This paperfocuses on the design of gain-scheduledcontrollersusing linear, parameter-varying (LPV) control techniques. LPV gainscheduled controllers directly incorporate guaranteed stability and performance levels in the control design process in contrast to the two approaches to multivariableengine control just cited. LPV gainscheduled controllers are obtained directly as part of the design process described in this paper, as opposed to conventional processes, where the gain schedules are developed after the fact to connect point designs.

The turbofan engine is modeled as an LPV system, and recent LPV synthesis results ${ }^{10,11}$ are applied to design a gain-scheduled, LPV controller that works well over a wide range of power codes. Seven linear, time-invariant (LTI) models are used in the control design, and it is assumed that the engine dynamics vary continuously in a linear manner between these seven points. This results in an LPV controllerconsisting of seven LTI controllers that are then gain scheduled via linear interpolation. A model matching approach is employed, such that the resulting closed loop resembles a decoupled set of second-ordersystems with specified rise times and overshoots. Specifically, we desire decoupled responses from reference inputs to fan speed, core engine pressure, and the overall engine pressureratio. The performance of linear $\mathcal{H}_{\infty}$ point designs (one at each power code) are compared with that of the LPV controllerat fixed operating points. A nonlinearsimulation is performed with the turbofanengine and the LPV controller varying as a function of power code. The LPV controller exhibits excellent tracking of reference commands as power code varies in time.

The engine and actuator models used in this paper correspond to the intelligent engine control (IEC) engine and can be found in Refs. 3 and 12 . Reference 3 examines the ability of single $\mathcal{H}_{\infty}$ point designs to cover a range of operating conditions at sea level. The models contain three states (two associated with the rotating compressor and fan and one lumped temperature); three inputs (fuel flow $W F$, exhaust nozzle area $A 8$, and variable bypass duct area $A 16$ ); and three outputs [corrected fan speed (PCN2R), core engine pres- sure ratio (CEPR), and overall engine pressure ratio (LEPR)]. Also see Ref. 8 for more detail regarding the engine inputs and outputs.

The paper is organized as follows. In Sec. II we briefly review the LPV synthesis procedure. The system interconnectionemployed for control designs is described in Sec. III. Section IV presents the results of several linear point designs, and Sec. V compares these results with those of the final LPV controller. Results of a nonlinear simulation using the LPV controller are presented in Sec. VI. We conclude with a brief summary of the results.

\section{LPV Gain Scheduling}

We begin with a brief introduction to gain scheduling based on linear parameter-varyingrepresentations. For a compact subset $\mathcal{P} \subset$ $\mathcal{R}^{s}$, the parameter variation set $\mathcal{F}_{\mathcal{P}}$ denotes the set of all piecewise continuous functions mapping $\mathcal{R}$ (time) into $\mathcal{P}$ with a finite number of discontinuities in any interval. A compact set $\mathcal{P} \subset \mathcal{R}^{s}$, along with continuous functions $A: \mathcal{R}^{s} \rightarrow \mathcal{R}^{n \times n}, B: \mathcal{R}^{s} \rightarrow \mathcal{R}^{n \times n_{d}}$, $C: \mathcal{R}^{s} \rightarrow \mathcal{R}^{n_{e} \times n}$, and $D: \mathcal{R}^{s} \rightarrow \mathcal{R}^{n_{e} \times n_{d}}$, represent an $n$th order LPV system, whose dynamics evolve as

$$
\left[\begin{array}{c}
\dot{\boldsymbol{x}}(t) \\
\boldsymbol{e}(t)
\end{array}\right]=\left[\begin{array}{ll}
A[\boldsymbol{\rho}(t)] & B[\boldsymbol{\rho}(t)] \\
C[\rho(t)] & D[\boldsymbol{\rho}(t)]
\end{array}\right]\left[\begin{array}{l}
\boldsymbol{x}(t) \\
\boldsymbol{d}(t)
\end{array}\right]
$$

where $\rho \in \mathcal{F}_{\mathcal{P}}$ (Ref. 13). The induced $\mathcal{L}_{2}$ norm of a quadratically stable LPV system $G_{\mathcal{F}_{\mathcal{P}}}$, with zero initial conditions, is defined as

$$
\left\|G_{\mathcal{F}_{\mathcal{P}}}\right\| \doteq \sup _{\rho \in \mathcal{F}_{\mathcal{P}}} \sup _{\|d\|_{2} \neq 0 d \in L_{2}} \frac{\|\boldsymbol{e}\|_{2}}{\|\boldsymbol{d}\|_{2}}
$$

This quantity is always finite. The quadratic LPV $\gamma$-performance problem is now stated. We assume that the controller has the form

$$
\left[\begin{array}{c}
\dot{\boldsymbol{x}}_{K}(t) \\
\boldsymbol{u}(t)
\end{array}\right]=\left[\begin{array}{ll}
A_{K}[\rho(t)] & B_{K}[\rho(t)] \\
C_{K}[\rho(t)] & D_{K}[\rho(t)]
\end{array}\right]\left[\begin{array}{c}
\boldsymbol{x}_{K}(t) \\
\boldsymbol{y}(t)
\end{array}\right]
$$

where $\rho \in \mathcal{F}_{\mathcal{P}}$ and $x_{K}$ represents the $m$-dimensionalcontrollerstate, and that the plant state-space representation is partitioned as

$$
\left[\begin{array}{c}
\dot{\boldsymbol{x}}(t) \\
\boldsymbol{e}(t) \\
\boldsymbol{y}(t)
\end{array}\right]=\left[\begin{array}{ccc}
A & B_{1} & B_{2} \\
C_{1} & D_{11} & D_{12} \\
C_{2} & D_{21} & D_{22}
\end{array}\right]\left[\begin{array}{l}
\boldsymbol{x}(t) \\
\boldsymbol{d}(t) \\
\boldsymbol{u}(t)
\end{array}\right]
$$

where $\boldsymbol{y}$ is the measurement, $\boldsymbol{u}$ is the control input, $\boldsymbol{d}$ is the exogenous disturbance, and $\boldsymbol{e}$ is the error output. The dependence of $A, B, C$, and $D$ on the parameter $\rho$ has been suppresseddue to space limitations.

Definition 1. Given a compact set $\mathcal{P} \subset \mathcal{R}^{s}$, a performance level $\gamma>0$, and the open-loop LPV plant in Eq. (3), the quadratic LPV $\gamma$-performance problem is solvable if there exists an integer $m \geq 0$, a matrix $W \in \mathcal{R}^{(n+m) \times(m+n)}, W=W^{T}>0$, and continuous bounded matrix functions $\left(A_{K}, B_{K}, C_{K}, D_{K}\right): \mathcal{R}^{s} \rightarrow\left(\mathcal{R}^{m \times m}\right.$, $\left.\mathcal{R}^{m \times n_{y}}, \mathcal{R}^{n_{u} \times m}, \mathcal{R}^{n_{u} \times n_{y}}\right)$ such that

$$
\left[\begin{array}{ccc}
A_{c}^{T} W+W A_{c}^{T} & W B_{c} & \gamma^{-1} C_{c}^{T} \\
B_{c}^{T} W & -I_{n_{d}} & \gamma^{-1} D_{c}^{T} \\
\gamma^{-1} C_{c} & \gamma^{-1} D_{c} & -I_{n_{e}}
\end{array}\right]<0
$$

for all $\rho \in \mathcal{P}$, where the matrices $A_{c}, B_{c}, C_{c}$, and $D_{c}$ are the closedloop state-space data that depend on $\rho$.

If there exists a controller such that Eq. (4) is satisfied, then the closed-loop system is quadratically stable and the induced $\mathcal{L}_{2}$ norm from $\boldsymbol{d}$ to $\boldsymbol{e}$, as defined in Eq. (2), is less than $\gamma$. The main result is that the existence of a controller that solves the quadratic LPV $\gamma$-performanceproblemcan be expressedas the feasibility of a set of affine matrix inequalities (AMIs), which can be solved numerically. For more details on LPV synthesis results the reader is referred to Refs. 11 and 14-16. The parameter $\rho$ is assumed to be available in real time, and hence, it is possible to construct an LPV controller whose dynamics adjust according to variations in $\rho$ and maintain stability and performance along all parameter trajectories.

This approach allows gain-scheduled controllers to be treated as a single entity, with the gain scheduling achieved via the parameterdependent controller. This allows for a simple implementation of 
the LPV controller, linear interpolation between the corresponding parameter-dependentstate-space controllermatrices. This approach has been successfullyapplied to the synthesis of missile autopilots, ${ }^{17}$ controllers for turbofan engines, ${ }^{18}$ and flight controllers. ${ }^{19}$

\section{Control Problem Formulation}

The system interconnectioninitially consideredfor controldesign is shown in Fig. 1. In Fig. 1, $P_{\text {sys }}$ represents the three-input, threeoutput, three-state model provided by Frederick et al. ${ }^{3}$ The $A, B, C$, and $D$ matrices vary as a function of power code (PC), with data provided for seven distinct power codes $P C=20,25, \ldots, 50$. The input and output units have been normalized. As already mentioned, inputs to the plant, $P_{\text {sys }}$, are fuel flow rate $W F$, nozzle area $A 8$, and bypass duct area $A 16$. Outputs are PCN2R, CEPR, and LEPR.

The problem is formulated as a model matching problem in the $\mathcal{H}_{\infty}$ and LPV framework. We desire the engine to respond as three single input/single output (SISO) systems with no off-diagonalcoupling. $P_{\text {mod }}$ represents such a decoupled system, and any difference between this desired model and the true plant is penalized via the weight $W_{p}$

The disturbance vector $\boldsymbol{d}_{1}$ represents reference commands to the inner-loopcontroller, whose values we wish the plant output to track. Specifically, these commands correspond to PCN2R $\mathrm{ref}_{\text {ref }}, \mathrm{CEPR}_{\mathrm{ref}}$, and LEPR $R_{\text {ref }}$. The disturbance vector $\boldsymbol{d}_{2}$ represents noise or actuator error, to which the controller should be robust. In the final control designs, this disturbance was moved to the plant output to facilitate designs with reduced-order weights (Fig. 1) for reasons explained in the following paragraphs.

By keeping the error vector $\boldsymbol{e}_{1}$ small, good tracking of $\boldsymbol{d}_{1}$ is ensured. The role of $\boldsymbol{e}_{2}$ is to penalize control effort, in terms of actuator magnitudes as well as actuator rates. The controller sees $\boldsymbol{y}_{c}$ as its input measurements, and generates $\boldsymbol{u}_{c}$ as its output control commands.

The actuator model $P_{\text {act }}$ taken from Ref. 3 is a diagonal augmentation of first-order lags with unity gain at DC gain,

$$
P_{\text {act }}=\left[\begin{array}{ccc}
26 /(s+26) & 0 & 0 \\
0 & 18 /(s+18) & 0 \\
0 & 0 & 29 /(s+29)
\end{array}\right]
$$

The actuator model shown in Fig. 1 supplies actuator positions $\boldsymbol{u}$ and actuator rates $\dot{\boldsymbol{u}}$, to allow actuator rates to be penalized in the control design. In our studies, a zero was added to the actuator position model at $1000 \mathrm{rad} / \mathrm{s}$, that is, $(0.001 s+26) /(s+26)$, to prevent the actuator penalty weights from rolling off to zero at high frequency. This is necessary because the LPV and $\mathcal{H}_{\infty}$ output feedback algorithms used for design require that all controls signals have a nonzero penalty at all frequencies. ${ }^{14,20}$ The control signals correspond to fuel flow $W F$, exhaust nozzle area $A 8$, and variable bypass duct area $A 16$ in this application and are represented by $\boldsymbol{u}_{c}$ in Fig. 1. This allows us to remove the rate bounds from the control problem (thus penalizing only the actuator positions) if desired, to study their role in the control design.

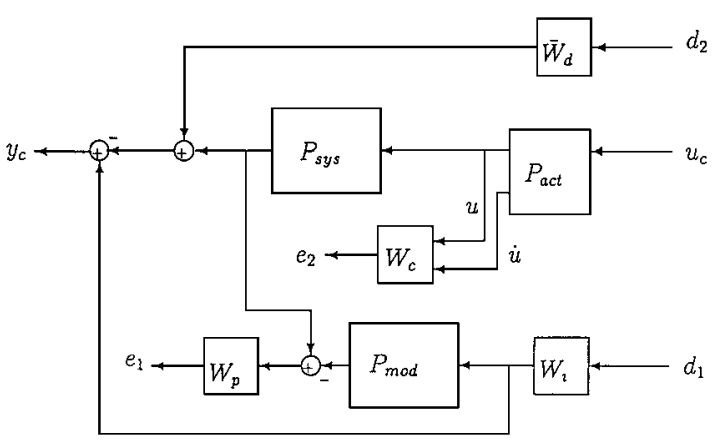

Fig. 1 Interconnection for control design.
The model matching block $P_{\bmod }$ was inferred from bandwidthand overshoot requirements specified in Ref. 3. The desired response is modeled as a second-ordersystem with unity gain at low frequency, a damping ratio $\xi=0.65$, and natural frequency $\omega_{0}=5.5 \mathrm{rad} / \mathrm{s}$,

$$
P_{\mathrm{mod}}=\frac{1}{\left(s / \omega_{0}\right)^{2}+\left(s / 2 \xi \omega_{0}\right)+1} I_{3 \times 3}
$$

It is desired to have the three transfer functions, $\mathrm{PCN} 2 \mathrm{R}_{\text {ref }} \rightarrow$ PCN2R, CEPR ref $_{\rightarrow}$ CEPR, and LEPR ref $_{\rightarrow}$ LEPR, have the same decoupled response characteristics. The input weight $W_{i}$ is a constant weighting used to normalize the inputs. The model in Ref. 3 was normalized such that a unit step in any input variable corresponds to a $10 \%$ change in the unscaled variable. As the authors in that work considered steps inputs of magnitude 5, we have chosen the input weight $W_{i}=\frac{1}{5} I_{3 \times 3}$. The $\mathcal{H}_{\infty}$ control design objective is to keep the $\mathcal{H}_{\infty}$ norm less than 1 . Note that the product of a control signal of magnitude 5 with the input weight is 1 . Hence, a $\mathcal{H}_{\infty}$ norm of the closed-loop system less than 1 implies that the control signals are less than 5 in magnitude.

The disturbance $W_{d}$ is used to model actuator errors as well as limit the bandwidth of control effort by ramping up at high frequency. For the disturbance to reach the controller, the weight $W_{d}$ must increase faster than the plant $P_{\text {sys }}$ rolls off, and this requires additional states in the interconnection. Moreover, the weight $W_{d}$ would possibly need to vary with PC, as the plant gains change significantly across PC. To simplify the LPV gain-scheduled control design, we chose to move the disturbance to the plant output (as shown in Fig. 1), where a first-order weight was sufficient to limit controllerbandwidth. We shall denote the output disturbance weight by $\bar{W}_{d}$,

$$
\bar{W}_{d}=0.35 \frac{s+0.03}{s+20} I_{3 \times 3}
$$

The penalties on the control deflections and rates, $W_{c}$, are selected to be constant as in Ref. 3 . Here we have chosen to penalize the normalized actuator movement larger than unity and normalized actuator rates larger than 10 in real units:

$$
W_{c}=\left[\begin{array}{cc}
I_{3 \times 3} & 0 \\
0 & 0.1 I_{3 \times 3}
\end{array}\right]
$$

The performance weight $W_{p}$ penalizes the difference between the desired ideal model and the actual closed-loop response of the turbofan engine. The larger is the magnitude of $W_{p}$, the smaller is the allowable difference between desired and actual output. In our designs, $W_{p}$ is a first-order low-pass weight given by

$$
W_{p}=\frac{s+900}{10 s+1} I_{3 \times 3}
$$

At low frequency (below $0.1 \mathrm{rad} / \mathrm{s}$ ), the $W_{p}$ weight corresponds to dc errors of $\frac{1}{900}$ or $0.11 \%$ tracking error. At $2-6 \mathrm{rad} / \mathrm{s}$, an error of between 2 and $6 \%$ is allowed between desired and actual response. At high frequency, the mismatch between actual and desired output is not penalized. Hence, this ensures good tracking of the response models in the bandwidth 2-6 rad/s, with little or no DC error.

\section{Linear Point Designs}

In this section we present several linear $\mathcal{H}_{\infty}$ point designs based on the interconnectionstructure in Fig. 1. The control objectives are as follows.

1) Demonstrate that a single (nominal) $\mathcal{H}_{\infty}$ controller does not achieve the desired closed-loop responses for the seven power code points.

2) Demonstrate that multiple $\mathcal{H}_{\infty}$ controllers (one for each plant) achieve the desired performance objectives.

3) Motivate an LPV design.

Seven $\mathcal{H}_{\infty}$ controllers were synthesized, one for each plant, using the interconnection of Fig. 1. The $\mathcal{H}_{\infty}$-norm achieved for these 

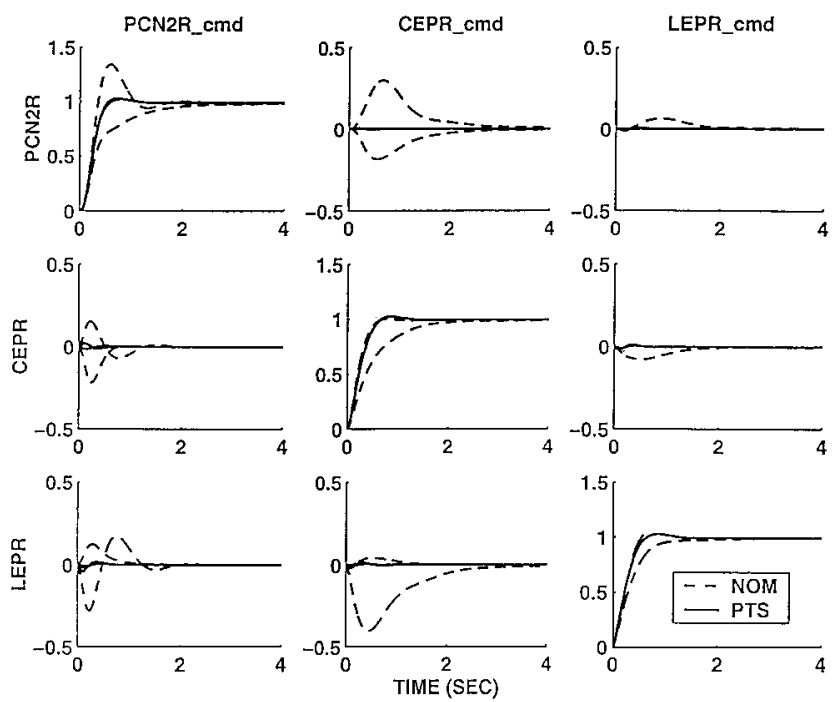

Fig. 2 Step responses of the turbofan engine at $\mathrm{PC}=20,35$, and 50 using a single $\mathcal{H}_{\infty}(\mathrm{PC}=35)$ controller $(---)$ and $\mathcal{H}_{\infty}$ point design controllers at each $\mathbf{P C}$.

designs ranged between 1.5 and 2.1. As the interconnection used has 18 states, so do each of the seven controllers. In this study, no attempt is made to reduce the order of the controllers.

Two approaches were considered to evaluate performance of the control designs. One involves a measure of the $\mathcal{H}_{\infty}$ norm from $\boldsymbol{d}$ to $\boldsymbol{e}$, which we desire to be smaller than 1, though values of as large as 2-5 were considered acceptable. The second judgment was made in terms of our specified objective, through inspection of the nominal step response of the closed-loop system. Tracking error, decoupling, and magnitude of actuator positions and rates were all evaluated in terms of our original objectives.

Figure 2 shows the amount of coupling present in the seven LTI models when using a single $\mathcal{H}_{\infty}$ controller designed at $\mathrm{PC}=35$ is used. (This operating point was chosen as the nominal to minimize the coupling over all PCs when using a single $\mathcal{H}_{\infty}$ controller.) The nine plots in Fig. 2 correspond to the three command inputs, PCN2R $R_{\text {ref }}, C E P R_{\text {ref }}$, and $L_{E P R}$ ref, to the outputs of the engine model PCN2R, CEPR, and LEPR. The control design objective was for each channel to have completely decoupled unity tracking with a second-order response.

Note that the step response in PCN2R varies dramatically as PC is varied using the controller designed for $\mathrm{PC}=35$. The off-diagonal terms (which we desire to be zero, based on the decoupled form of our model in the model matching approach) are on average of size 0.2. The largest coupling is seen in the step response from $\mathrm{CEPR}_{\text {ref }}$ to LEPR, which has a peak of roughly 0.45 at $\mathrm{PC}=20$. Thus, we see that the closed-loop system is decoupled at the PC for which the controller was designed, but shows significant coupling at other PCs. The same is true of our desired tracking response, which is good near the nominal model, but exhibits overshoot (for $\mathrm{PC}=20$ ) and a slow response time (for PC $>40$ ) at other power codes.

Consider a linear $\mathcal{H}_{\infty}$ controller synthesized at each PC for the turbofan engine. The solid line responses in Fig. 2 demonstrate the ability of individual $\mathcal{H}_{\infty}$ controllers (one designed for each PC) to achieve the desired result. Note the decrease in coupling, together with the desired step response in the diagonal elements. Here the coupling terms are at least a factor of 10 smaller than the single $\mathcal{H}_{\infty}$ point design at $\mathrm{PC}=35$. This is typical of the other six operating point time responses. The actuator magnitudes never exceeded 3 for unit step commands (see the dashed lines in Fig. 3), and the actuator rates remained between \pm 15 units/s of the scaled input signals. Notice that these numbers are larger than the limits we originally specified in our problem setup, and indicate values of $\gamma>1$ in the $\mathcal{H}_{\infty}$ synthesis.

Loop-at-a-time gain and phase margins were calculated, and all of the $\mathcal{H}_{\infty}$ point designs achieve at least $14 \mathrm{~dB}$ of gain margin at a frequency of $13.8 \mathrm{rad} / \mathrm{s}$ and $64 \mathrm{deg}$ of phase margin at $3.7 \mathrm{rad} / \mathrm{s}$. The
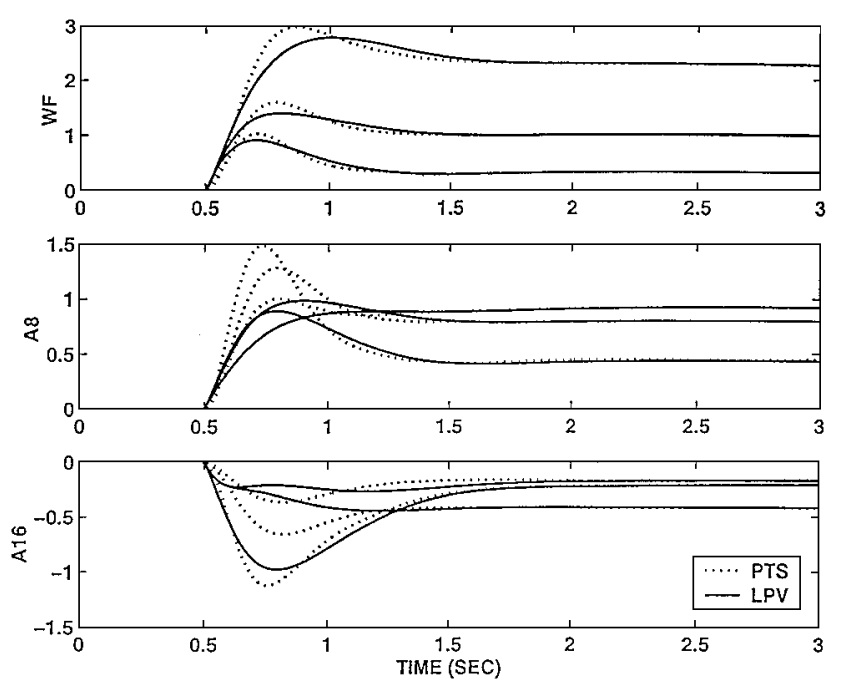

Fig. 3 Linear actuator responses for a unit step in commanded PCN2R ref.
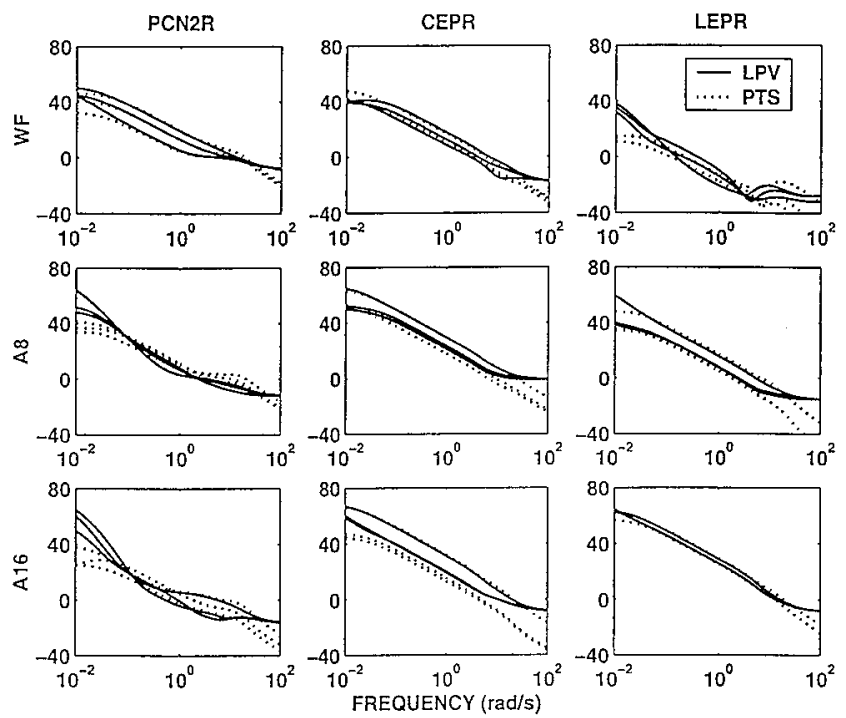

Fig. 4 Controller magnitude plots for $P C=20,35$, and 50; $\cdots, \mathcal{H}_{\infty}$ designs and - LPV point controllers.

dashed lines in Fig. 4 show magnitude plots of the $\mathcal{H}_{\infty}$ point designs at $\mathrm{PC}=20,35$, and 50. Note how the response of these controllers changes as a function of PC. Also of interest is a comparison between the $\mathcal{H}_{\infty}$ and LPV designs; the differences, though not drastic, are apparent.

\section{LPV Control Design}

The $\mathcal{H}_{\infty}$ controllers designed at each PC achieve the desired performance and robustness objectives on the corresponding turbofan model. The problem is that there is no systematic technique for scheduling these controllers as a function of PC. Moreover, there are no guarantees that these scheduled controllers would stabilize the engine for all PCs and achieve the performance objectives. LPV control design techniques, on the other hand, are formulated to synthesize a scheduled controller for all PCs that guarantees stability and performance throughout the engine operating regime.

The LPV controller in this work was synthesized using the interconnection in Fig. 1, together with the seven plant models. The same weighting functions used in the $\mathcal{H}_{\infty}$ point designs are used in the LPV design, though the engine model varies as a function of PC. By using the same interconnection and weights, we obtain an LPV controller that meets the same performance and robustness objectives as our original $\mathcal{H}_{\infty}$ point designs.

The LPV controller measures the errors in PCN2R, CEPR, and LEPR responses and schedules on PC. (In practice one might 

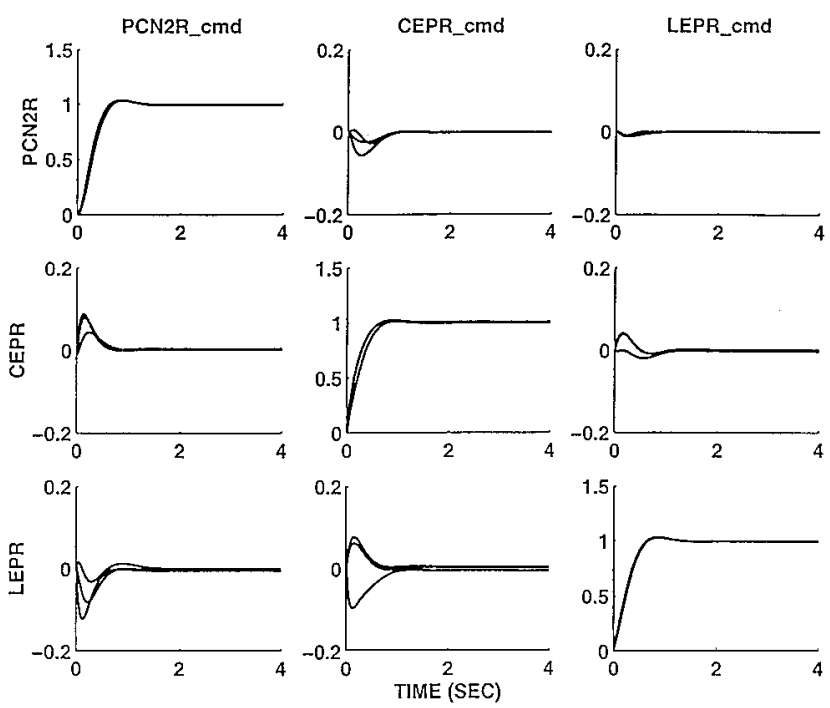

Fig. 5 Step responses for seven plant models using a single LPV controller.

schedule on PCN2R or a lagged version of PC, reflecting the fact that the engine dynamics do not change instantly with changes in PC. Because our models are parametrized by PC, however, we shall consider the controller to be parametrized by PC as well, independent of what is actually used to schedule the engine in practice.) The synthesis technique requires solving an linear matrix inequality (LMI) over the entire parameter space for which there are seven points. Recall from the $\mathcal{H}_{\infty}$ point designs, the $\mathcal{H}_{\infty}$ norm varied from 1.5 to 2.1 across PC. For the LPV control design, the induced $\mathcal{L}_{2}$ norm for all parameter trajectories was 2.45 . This norm is only slightly larger than the maximum value associated with the seven point designs, which indicates that the performance and robustness of the gain-scheduledLPV design should be close to that of the linear $\mathcal{H}_{\infty}$ point designs. As with the point designs, the LPV controller has 18 states. No attempt is made to reduce the order of the controller.

Figure 5 shows step response results using a single LPV controller. Note that decoupling is much better than with the single nominal controller, though not quite as good as that of individual point designs(Fig. 2). (The point designs represent the best we could hope to do for frozen parameter values.) In contrast, however, the performance of the LPV controller is guaranteed not only at the design points, but at intermediate values of PC as well. The LPV controller should perform as well in the case when PC is changing. Moreover, implementation of the gain-scheduled LPV controller requires only linear interpolation of the state-space data. The LPV design presented does not use any rate information in the synthesis of controllers. If bounds were available on how fast the PCs were allowed to vary, this information could also be included into the LPV synthesis to obtain a more aggressive controller. The present design is conservative in that it allows for (or defends against) infinitely fast variation in PC.

For the LPV point responses to unit step commands, the actuator magnitudes never exceeded 3 for unit step commands (see the solid lines in Fig. 3), and the actuator rates remained between \pm 10 units/s of the scaled input signals. Here we are primarily concerned with maintaining similar actuator rates and bounds as we move from $\mathcal{H}_{\infty}$ point designs to an LPV controller.

Loop-at-a-time gain and phase margins were calculated, and the LPV point controller achieve at least $28 \mathrm{~dB}$ of gain margin at a frequency of $27 \mathrm{rad} / \mathrm{s}$ and $65 \mathrm{deg}$ of phase margin at $3.6 \mathrm{rad} / \mathrm{s}$. In practice, it is often the case that the non-rate-boundedLPV controllers have increased robustness as compared with the corresponding $\mathcal{H}_{\infty}$ point design. However, this increased robustness comes at the expense of the system performance, for example, decoupling in this case. A magnitude plot of the LPV point controllers at PC $=20,35$, and 50 are shown with solid lines in Fig. 4. Note how the response of LPV point controllers change as a function of PC and have similar magnitude characteristics to the corresponding $\mathcal{H}_{\infty}$ point designs at the same power levels.

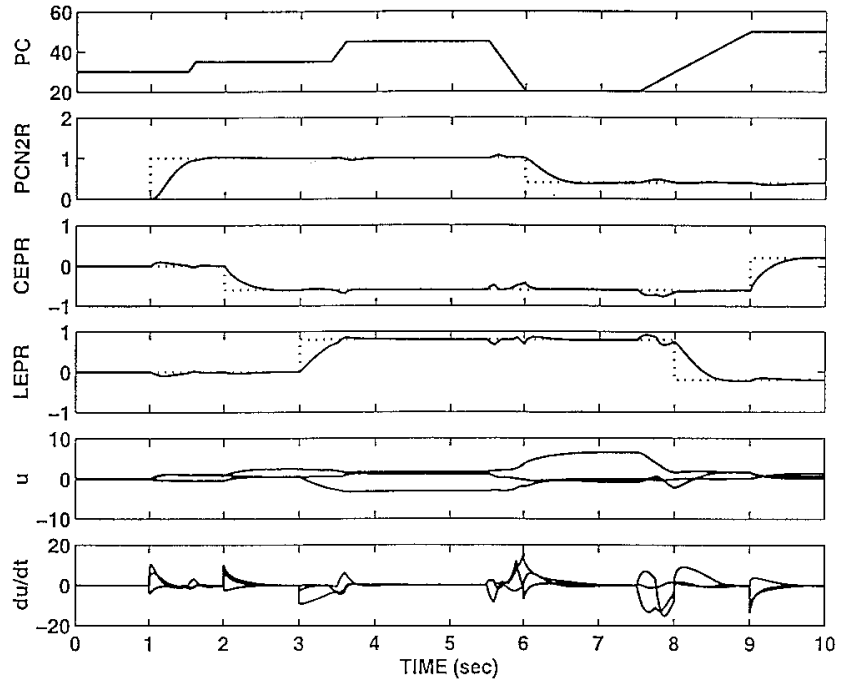

Fig. 6 Nonlinear simulation of turbofan with the LPV controller; $\cdots \cdot$, reference commands and $\_$, closed-loop response.

\section{LPV Simulation}

To further test and compare the LPV controller with $\mathcal{H}_{\infty}$ designs, a simulation was performed in which reference command steps were applied to the system while the PC varies as a function of time. The top plot of Fig. 6 shows the PC trajectory, which covers the range of values from 20 to 50 . The nonlinear simulation is performed with the PC varying as a function of time. The variation in PC and the reference command inputs used in this simulation are not necessarily representative of normal changes in operating conditions, but rather are intended to illustrate the ability of the LPV controller to track the reference inputs during large and abrupt changes in PC. The LPV controller is gain scheduled as a function of PC via linear interpolation. Figure 6 shows simulation results using the LPV controller. The LPV controllers is designed specifically to be implemented via linear interpolation. The reference commands to PCN2R, CEPR, and LEPR are shown as dashed lines in the plots, and the closedloop responses are the solid lines. The tracking performance of the LPV controller is very close to the desired response with only small deviations in tracking of the LEPR reference command. The maximum tracking error seen is 0.23 (recall all signals are normalized to unit magnitude). If we define the model matching error to be the difference between the desired model output and the actual closedloop model output (with the LPV controller in place), the energy in the model matching error relative to that of the desired model output is 6.53 .

It is also of interest to examine the actuator position and rate response for the LPV simulation. As in the case of the linear unit step responses, the actuator magnitude responses remain within the \pm 5 unit limits, and the actuator rates remain below \pm 15 units/s.

\section{Conclusions}

Recent advancesin robust control synthesisfor LPV systems have been applied to a model of a turbofan engine. The control objective was to decouple the multi-input/multi-outputsystem into three independent channels with minimal cross coupling, subject to actuator magnitude and rate limitations. $\mathcal{H}_{\infty}$ point designs provided an initial starting point, giving an indication of the performance that might be expected of an LPV controller. After obtaining reasonable point designs, an LPV controller was synthesized and its performance evaluated.

In a parameter-varying simulation, it was shown that the LPV controller provides excellent tracking response and does not exceed actuator magnitude and rate constraints. Although synthesis of the LPV controller is computationally more intensive than synthesis of linear point designs ( 2 min to compute all seven $\mathcal{H}_{\infty}$ point designs, 45 min to arrive at the LPV controller on a $300-\mathrm{MHz}$, Pentium II machine with 64-MB RAM), LPV synthesis techniques design a global controller for all values of the scheduled variable that guarantees 
both stability and performance in the presence of infinitely fast time variations of the scheduled variable. Although presented here for a single scheduling variable, LPV methods can also be applied to the case of multiple scheduling variables.

Summarizing, the gain-scheduled LPV design approach described is well suited to the design of inner loops for turbofan engines, is computationally tractable, and provides performance guarantees under rapid changes in the scheduling variables.

\section{Acknowledgments}

This work was funded by NASA John H. Glenn Research Center at Lewis Field, Contract NASA/NAG-3-1975, with JohnathanLitt as the Contract Monitor. The authors would like to thank Dean Frederick for supplying the turbofan engine models, Sanjay Garg for many useful discussion on turbofan engine control, and the reviewers for their helpful comments.

\section{References}

${ }^{1}$ Kandebo, S. W., "GE Eyes Variable Cycle for Engine Competition," Aviation Week and Space Technology, Vol. 144, No. 13, 1993, pp. 42, 43.

${ }^{2}$ Adibhatla, S., "Propulsion Control Law Design for the NASA STOVL Controls Technology Program," Proceedings of the AIAA International Powered Lift Conference, AIAA, Washington, DC, 1993, pp. 271-280.

${ }^{3}$ Frederick, D. K., Garg, S., and Adibhatla, S., "Turbofan Engine Control Design Using Robust Multivariable Control Technologies," AIAA Paper 96-2587, July 1996.

${ }^{4}$ Merrill, W., Lehtinen, B., and Zeller, J., "The Role of Modern Control Theory in the Design of Controls for Aircraft Turbine Engines," Journal of Guidance, Control, and Dynamics, Vol. 7, No. 6, 1984, pp. 652-661.

${ }^{5}$ Nordgren, R. E., Gastineau, Z., Adibhatla, S., Grewal, G. S., and Nwokah, O. D. I., "Robust Multivariable Turbofan Engine Control: A Case Study," Proceedings of the 33rd IEEE Control and Decision Conference, IEEE Publications, Piscataway, NJ, 1994, pp. 1086-1092.

${ }^{6}$ Edmunds, J. M., "Control System Design and Analysis Using ClosedLoop Nyquist and Bode Arrays," International Journal of Control, Vol. 30, No. 5, 1979, pp. 773-802.

${ }^{7}$ Shamma, J., and Athans, M., "Gain Scheduling: Potential Hazards and Possible Remedies," Proceedings of the 1991 American Control Conference, IEEE Publications, Piscataway, NJ, 1991, pp. 516-521.
${ }^{8}$ Polly, J. A., Adibhatla, S., and Hoffman, P. J., "Multivariable Turbofan Engine Control for Full Flight Envelope Operation," International Gas Turbine and Aeroengine Congress and Exposition, ASME-88-GT-6, American Society of Mechanical Engineers, Fairfield, NJ, 1998, pp. 541-548.

${ }^{9}$ Watts, S. R., and Garg, S., "A Comparison of Multivariable Control Design Techniques for a Turbofan Engine Control," International Gas Turbine and Aeroengine Congress and Exposition, ASME-95-GT-258, American Society of Mechanical Engineers, Fairfield, NJ, 1995, pp. 307-314.

${ }^{10}$ Packard, A., "Gain Scheduling via Linear Fractional Transformations," Systems and Control Letters, Vol. 22, No. 2, 1994, pp. 79-92.

${ }^{11}$ Packard, A., and Kantner, M., "Gain Scheduling the LPV Way," Proceedings of the 35th IEEE Control and Decision Conference, IEEE Publications, Piscataway, NJ, 1996, pp. 3938-3941.

${ }^{12}$ Adibhatla, S. H., Brown, H., and Gastineau, Z., "Intelligent Engine Control (IEC)," AIAA Paper 92-3484, July 1992.

${ }^{13}$ Kamen, E., and Khargonekar, P., "On the Control of Linear Systems Whose Coefficients are Functions of Parameters," IEEE Transactions on Automatic Control, Vol. AC-29, No. 1, 1984, pp. 25-33.

${ }^{14}$ Becker, G., "Quadratic Stability and Performance of Linear Parameter Dependent Systems," Ph.D. Dissertation, Dept. of Mechanical Engineering, Univ. of California, Berkeley, CA, 1993.

${ }^{15}$ Shahruz, S. M., and Behtash, S., "Design of Controllers for Linear Parameter-Varying Systems by the Gain Scheduling Technique," Proceedings of the 29th IEEE Control and Decision Conference, IEEE Publications, Piscataway, NJ, 1990, pp. 2490-2491.

${ }^{16}$ Apkarian, P., and Gahinet, P., "A Convex Characterization of GainScheduled $H_{\infty}$ Controllers," IEEE Transactions on Automatic Control, Vol. AC-40, No. 5, 1995, pp. 853-864.

${ }^{17}$ Wu, F., Packard, A., and Balas, G. J., "LPV Control Design for PitchAxis Missile Autopilots," Proceedings of the 34th IEEE Control and Decision Conference, IEEE Publications, Piscataway, NJ, 1995, pp. 188-193.

${ }^{18}$ Wolodkin, G., Balas, G. J., and Garrard, W. L., "Application of Parameter-Dependent Robust Control Synthesis to Turbofan Engines," AIAA Paper 98-0973, Jan. 1998.

${ }^{19}$ Balas, G. J., Fialho, I. J., Packard, A. K., Renfroa, J. W, and Mullaney, C., "On the Design of LPV Controllers for the F-14 Lateral-Directional Axis During Powered Approach," in Proceedings of the 1997 American Control Conference, IEEE Publications, Piscataway, NJ, 1997, pp. 123-127.

${ }^{20}$ Doyle, J., Glover, K., Khargonekar, P., and Francis, B., "State-Space Solutions to Standard $\mathrm{H}_{2}$ and $\mathrm{H}_{\infty}$ Control Problems," IEEE Transactions on Automatic Control, Vol. AC-34, No. 8, 1989, pp. 831-847. 